\title{
Evaluación de la fuerza de doblado y de fricción en el conformado de chapa de acero inoxidable AISI 304 DDQ mediante ensayos de doblado en condiciones multiaxiales de embutición
}

\author{
J. Coello ${ }^{* * *}$, V. Miguel ${ }^{* * *}$, C. Ferrer ${ }^{* * *}$, A. Calatayud ${ }^{* * *}$ y A. Martínez
}

\begin{abstract}
Resumen El radio de entrada a la matriz se considera una de las zonas críticas en los procesos de conformado de chapa mediante embutición profunda. El análisis de la fuerza de fricción y de doblado existentes resulta importante para predecir el comportamiento de la chapa en dicha zona, así como para garantizar una lubricación adecuada a las condiciones de procesado. En el presente trabajo, se aplica un método de ensayo que evalúa las acciones en el proceso de doblado del acero AISI 304 DDQ bajo condiciones similares a las que sufre el material en los procesos de embutición y que no son reproducidas por los clásicos ensayos de doblado bajo tensión. Se establecen como variables la deformación experimentada previamente por el material en condiciones típicas de cortante puro, "pure shear", el ángulo de doblado y el radio de doblado. Los resultados obtenidos permiten establecer la presión de contacto herramienta-chapa, creciente con el ángulo de doblado y decreciente con el radio. Aunque dichos resultados guardan tendencias similares a los obtenidos de forma analítica, aconsejan introducir correcciones con el fin de conseguir mayor grado de concordancia del método analítico.
\end{abstract}

Palabras clave

Acero inoxidable AISI 304; Doblado bajo tensión; Fricción; Embutición.

\section{Friction and bending forces evaluation of AISI 304 DDQ steel sheet forming by bending tests under deep-drawing multiaxial stresses}

\begin{abstract}
Die radius is a critical area from the viewpoint of friction in forming processes. Moreover the sheet, that has been previously deformed in flange area, suffers bending and unbending stresses. Then, die-sheet contact in die radius must be especially considered in order to guarantee the suitable lubrication conditions. In the present work, a test method is carried out for evaluating an AISI 304 DDQ steel under similar conditions to those existing in the die radius area and that, usually, are not really reproduced in traditional bending under tensions tests. Deformation under pure shear condition, the bending and the radius angle have been established as variables of the tests. Results allow to obtain the apparent pressure sheet-bending tool, that increases with bending angle and decreases with tool radius. This last variable is the most significant while the bending angle has lesser influence. Although experimental results present some concordances with values obtained by analytical methods, some corrections must be considered in them in order to improve the theoretical values.
\end{abstract}

Keywords

AISI 304 Stainless steel; Bending under tension; Friction; Deep-drawing.

\section{INTRODUCCIÓN}

En los procesos de embutición profunda una de las etapas más complejas es el doblado del material de acuerdo al radio de la matriz, debido principalmente a las altas presiones alcanzadas en este punto. Para estudiar el comportamiento del material en esta zona se han desarrollado una gran variedad de ensayos de doblado bajo tensión, BUT, que incorporan diferentes geometrías, métodos de aplicación de carga, grado de deformación del material y velocidad de ensayo.

Uno de los métodos que ha recibido especial interés, y que ha sido utilizado por diversos

(•) Trabajo recibido el día 16 de enero de 2012 y aceptado en su forma final el día 11 de abril de 2012.

* Instituto de Desarrollo Regional; Universidad de Castilla-La Mancha; Avda. España s/n 02006 Albacete.

** Escuela de Ingenieros Industriales Albacete; Universidad de Castilla-La Mancha; Avda. España s/n 02006 Albacete.

*** Departamento de Ingeniería Mecánica y de Materiales; Universidad Politécnica de Valencia; Camino de Vera 46022 s/n. 
autores $^{[1}$ y 2], es el ensayo BUT realizado en dos etapas. En la primera etapa, el ensayo se realiza utilizando un cilindro fijo ( en la segunda etapa el ensayo se lleva a cabo en las mismas condiciones pero con un cilindro que gira libremente, asumiendo la no existencia de rozamiento en el giro del cilindro como hipótesis. De este modo, se evalúa la fuerza de doblado en la segunda etapa y la de rozamiento en el cilindro en la primera. El inconveniente fundamental que presenta este ensayo es que la determinación del rozamiento se efectúa de manera indirecta sin establecer el grado de implicación de la resistencia a la rodadura del cilindro móvil.

Hao et al..$^{[3]}$ simulan el comportamiento tribológico del proceso de embutición en el radio del punzón mediante ensayos de doblado bajo tensión, realizados sobre un dispositivo en $U$, alojado sobre una máquina universal de ensayos. Estos autores justifican que el dispositivo empleado simula el comportamiento a fricción de los procesos de embutición, determinando el coeficiente de fricción en la zona de doblado. Sin embargo, no consideran que el material en el proceso de embutición es sometido a un proceso de deformación en condiciones de multiaxialidad, en las que las presiones de contacto son muy superiores a las estudiadas en este trabajo.

A. Azushima et al. [4 y 5] estudian el comportamiento tribológico en la zona curva de la matriz de embutición mediante un dispositivo que evalúa directamente la fuerza de embutición y la tensión a tracción aplicada considerando que la distribución de presiones en la matriz es uniforme. Determinan la variación del coeficiente de rozamiento con la presión de contacto concluyendo que en régimen de lubricación límite el coeficiente de fricción permanece constante con la presión. Por el contrario, el coeficiente de fricción disminuye al pasar a un régimen de lubricación mixta. Aunque determinan el coeficiente de rozamiento en una sola etapa del ensayo, las tensiones de sujeción aplicadas son arbitrarias y el material no es sometido al proceso de tensiones multiaxiales típico de la embutición.

Saha y Wilson ${ }^{[6]}$ determinan analíticamente la influencia sobre el coeficiente de fricción de distintos factores que intervienen en los procesos de doblado bajo tensión. Los autores mencionados consideran de forma genérica una distribución uniforme de presión en la zona de doblado y un arco de contacto que coincide con el de doblado durante todo el ensayo. En este sentido, diversos autores han comprobado que estas hipótesis no se cumplen en todos los casos, principalmente en materiales con una elevada resistencia al doblado $^{[7-10]}$. Pereira et al. ${ }^{[7]}$ establecen una descripción cualitativa de la distribución de tensiones en la zona de contacto chapa-matriz durante el doblado bajo tensión indicando la existencia de picos de presión durante el contacto. Dichos autores comprueban la distribución planteada mediante el análisis de elementos finitos, estableciendo que la tensión de contacto es no uniforme ${ }^{[8]}$. Hanaki et al ${ }^{[9]}$.demuestran la existencia de picos de presión durante el doblado y desdoblado de una chapa, mediante la medida directa de la presión de contacto en el cilindro. Demuestran que a la entrada y salida de la matriz se producen sendos picos de presión, manteniéndose constante entre estas dos zonas. Coubrough et al ${ }^{[10]}$.determinan el ángulo y la distribución de presiones en la zona de contacto mediante un sensor piezoeléctrico colocado en el interior del cilindro de doblado. Mediante este estudio revelan que el ángulo de contacto de la chapa con la matriz es menor que el ángulo de doblado. El cilindro en estos ensayos es giratorio, por lo que los resultados obtenidos están referidos a ensayos realizados en ausencia de fricción y, por tanto, no se puede establecer de manera determinante que dichos valores no se modifiquen con la presencia de fricción.

Andreasen et al ${ }^{[11]}$. desarrollan un dispositivo de ensayos BUT que permite medir directamente la fuerza de fricción en el doblado sobre un cilindro fijo, determinando con gran precisión la influencia de los factores más importantes que afectan a la fricción. En este trabajo, al igual que en la mayoría de los estudios existentes en la literatura, la fuerza de tracción ejercida presenta valores inferiores al límite elástico, y por tanto no se produce deformación plástica en el material.

Miguel et al ${ }^{[12]}$. han desarrollado y validado un ensayo de doblado bajo tensión sobre acero DC-05, en el que el material es sometido simultáneamente a doblado y deformación en condiciones de tracción compresión-biaxial demostrando que el estado previo del material influye significativamente sobre el comportamiento tribológico del mismo en el proceso de doblado. Siguiendo la metodología iniciada por estos autores, en el presente trabajo se analiza el comportamiento tribológico en el radio de entrada a la matriz de embutición para el acero inoxidable AISI 304 DDQ, determinando la influencia de la presión, ángulo de doblado y radio de doblado en el proceso. Se determina la fuerza de doblado en el cilindro a partir de las distintas fuerzas que actúan en el sistema medidas experimentalmente, sin considerar ninguna hipótesis de fuerzas. 


\section{DESARROLLO EXPERIMENTAL}

\subsection{Materiales utilizados y variables estudiadas}

El material investigado en este trabajo es chapa de acero inoxidable austenítico AISI 304 DDQ de espesor, $\mathrm{t}, 0,8 \mathrm{~mm}$, con un acabado superficial comercial 2B (UNE EN ISO 10088-2). Dicho acabado se obtiene mediante un proceso de laminación en frío, recocido en atmósfera oxidante, decapado y con una pasada de temperizado (skin pass).

La composición química y las propiedades mecánicas del acero se indican en las tablas I y II. En la tabla II se establecen también los índices de anisotropía normal, r, y de endurecimiento, n, correspondientes al acero. La rugosidad media del acero es $\mathrm{R}_{\mathrm{a}} 0,08 \mu \mathrm{m}$.

\subsection{Ensayos de doblado bajo tensión}

El objeto de este ensayo es simular el comportamiento tribológico del acero inoxidable AISI 304 DDQ en la zona de doblado en el radio de entrada a la matriz durante los procesos de embutición profunda de chapa. La chapa es sometida a una deformación bajo condiciones de tracción-compresión biaxial, típicas de la acción existente bajo el prensachapas, e inmediatamente después es sometida al proceso de doblado. De este modo, la chapa es doblada tras haber sido sometida a un estado de cortadura pura o "pure shear" en el que la deformación se produce en el plano principal de la chapa, sin variación en el espesor de la misma ${ }^{[13]}$. Dada la sucesión de acciones que tienen lugar de forma simultánea durante el ensayo, la chapa es doblada bajo un estado tensional similar al que tiene lugar en el proceso de embutición de chapa ${ }^{[12]}$. En la figura 1 se indica el esquema de definición del ensayo.

La realización del ensayo se llevó a cabo mediante un dispositivo desarrollado experimentalmente y que permite variar la fuerza de cierre de la matriz con forma de cuña, $F_{\mathrm{N}}$, el ángulo de doblado, $\alpha$, y el radio de doblado, $\mathrm{R}_{\mathrm{C}}$. La matriz de deformación en forma de cuña, el prensachapas utilizado y el cilindro de doblado se han ejecutado en acero templado con una dureza 68 HRC. Una descripción más detallada del dispositivo mencionado puede encontrarse en los tra-

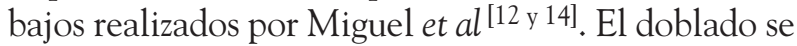
ejecuta en torno a un cilindro de radio conocido, $R_{d}$, sobre el que es posible medir la reacción, $\mathrm{F}_{\rho}$, en la bisectriz del ángulo de doblado.

Las probetas ensayadas se obtuvieron cortando bandas de chapa de acero AISI 304DDQ en estado de recepción, variando la anchura inicial entre 11,5 y $15 \mathrm{~mm}$. Para poder introducir las muestras en la matriz, se mecanizó un estrangulamiento con la forma de la matriz.

La velocidad de estirado de la chapa y la presión aparente en la matriz se consideraron como constantes de ensayo, fijando los correspondientes valores en $100 \mathrm{~mm} / \mathrm{min}$ y 3,90 MPa, respectivamente. Para mantener constante la presión de cierre en la matriz se selecciona una fuerza de cierre $\mathrm{F}_{\mathrm{N}}$ diferente, en función de la anchura de la muestra a ensayar, que

Tabla I. Composición química (\%) del acero AISI 304 DDQ

Table I. Chemical composition of AISI 304 DDQ steel

\begin{tabular}{ccccccccc}
\hline $\mathbf{C}$ & $\mathbf{S}$ & $\mathbf{S i}$ & $\mathrm{Mn}$ & $\mathbf{C r}$ & $\mathrm{Ni}$ & $\mathrm{Mo}$ & $\mathbf{V}$ & $\mathbf{C u}$ \\
\hline 0,040 & 0,003 & 0,19 & 1,11 & 17,67 & 9,01 & 0,28 & 0,08 & 0,26 \\
\hline
\end{tabular}

Tabla II. Propiedades mecánicas e índices de deformabilidad del acero AISI 304 DDQ

Table II. Mechanical properties and formability indices of AISI 304 DDQ steel

\begin{tabular}{cccccc}
\hline Acero & $\begin{array}{r}\mathbf{R}_{\mathrm{p0.2}} \\
(\mathrm{MPa})\end{array}$ & $\begin{array}{r}\mathbf{R}_{\mathrm{m}} \\
(\mathrm{MPa})\end{array}$ & HV0,5 & $\mathbf{R}$ & $\mathbf{n}$ \\
\hline 304 DDQ (2B) & 252 & 582 & 174 & 0,906 & 0,244 \\
\hline
\end{tabular}




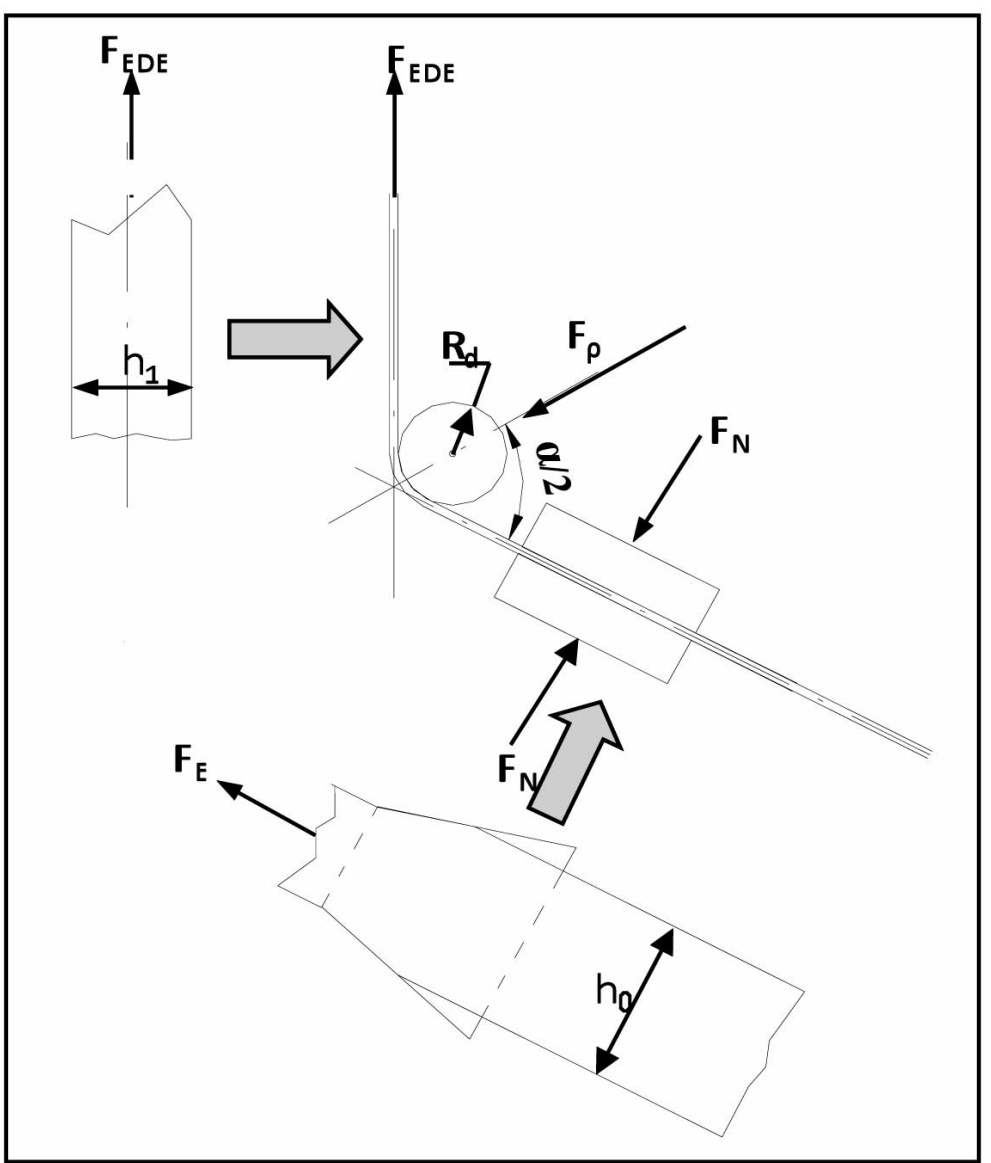

Figura 1. Esquema del ensayo de doblado y estirado[12 y 14$]$.

Figure 1. Diagram of bending under tensión test ${ }^{[12}$ and 14].

define una determinada superficie de contacto con la matriz, la cual es evaluada geométricamente.

Como lubricante se empleó una mezcla de aceite $/ \mathrm{S}_{2} \mathrm{Mo}$ al $50 \%$ en peso. Este tipo de lubricante garantiza la existencia de película de lubricante en condiciones elevadas de presión, dando lugar a un coeficiente de rozamiento sensiblemente inferior al que se obtiene mediante lubricación con aceites minerales ${ }^{[15]}$.

Las variables de ensayo consideradas son el ángulo de doblado, el radio de doblado y la deformación producida en el material. Se han considerado tres ángulos de doblado, $150^{\circ}, 120^{\circ}$ y $90^{\circ}$, y dos radios de doblado, $3,5 \mathrm{~mm}$ y $6,5 \mathrm{~mm}$, como representativos de los procesos de embutición. La deformación que se produce en el material, $\varepsilon$, se ha establecido mediante la deformación logarítmica que experimenta la anchura de la banda de chapa antes y después de su paso por la matriz de cuña, $h_{0}$ y $h_{1}$, respectivamente, conforme a la ecuación (1):

$$
\varepsilon=\ln \frac{\mathrm{h}_{1}}{\mathrm{~h}_{0}}
$$

\subsubsection{Determinación de la fuerza de rozamiento y de la fuerza de doblado en el cilindro}

Uno de los objetivos del ensayo de doblado bajo tensión definido previamente es la determinación de la fuerza de rozamiento, $F_{r c}$ y de doblado, $F_{d}$, en el cilindro, mediante un balance de las diferentes fuerzas que intervienen en el ensayo (Fig. 2).

A la entrada al cilindro la chapa está sometida a una fuerza, $\mathrm{F}_{\mathrm{E}}$, que es la fuerza necesaria para producir la deformación en la matriz de cuña con las condiciones de rozamiento correspondientes. A la salida del cilindro, la fuerza aplicada sobre la chapa es la fuerza total de estirado, $\mathrm{F}_{\mathrm{EDE}}$, medida directamente en el ensayo. De esta forma se cumple la relación que se establece en la ecuación (2), habida cuenta de que la fuerza de doblado $\mathrm{F}_{\mathrm{d}}$, se considera habitualmente en los términos de la fuerza necesaria en el sentido de estirado de la chapa para realizar el doblado y desdoblado de la misma ${ }^{[16]}$.

$$
F_{E D E}=F_{E}+F_{r C}+F_{d}
$$




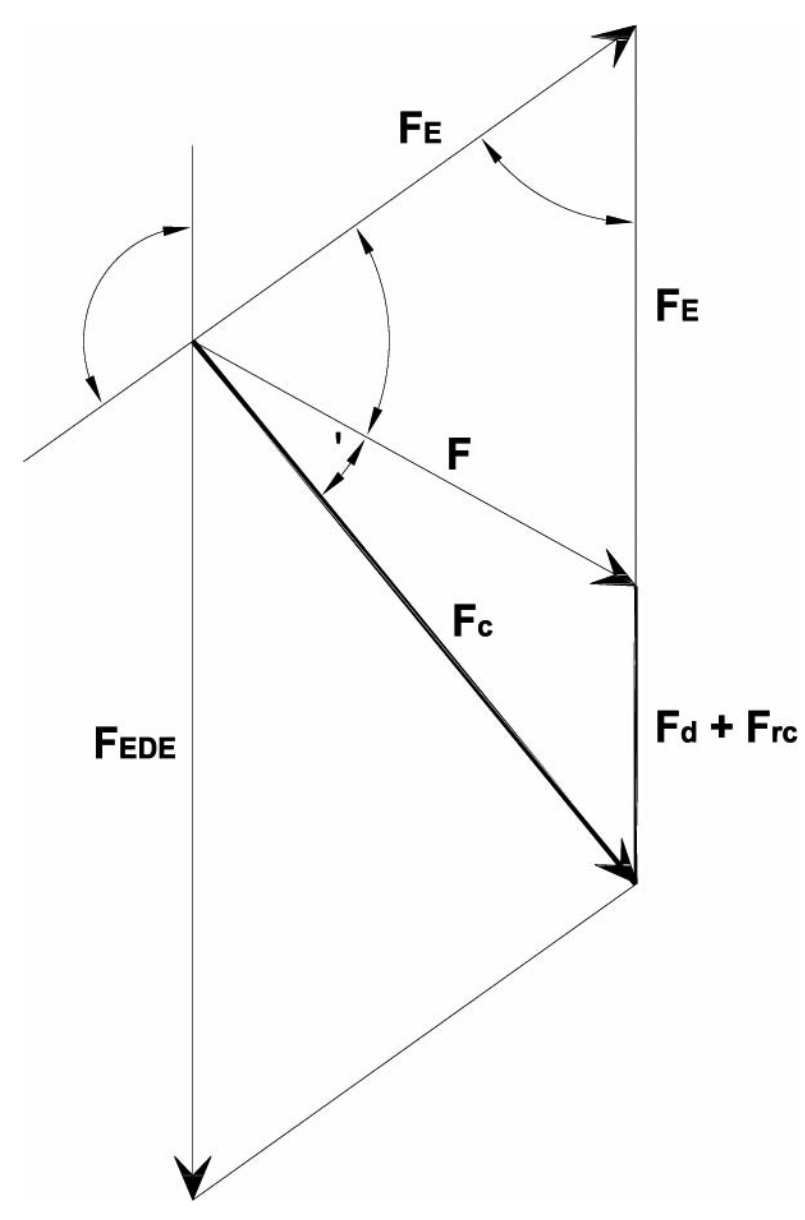

Figura 2. Diagrama vectorial de las fuerzas que intervienen en el ensayo de doblado.

Figure 2. Vectorial diagram of forces that take place in the bending test.

Los valores correspondientes a la fuerza de entrada al cilindro, $\mathrm{F}_{\mathrm{E}}$, se determinan en función de la anchura del material en trabajos previos bajo las condiciones indicadas ${ }^{[13]}$, por lo que es un valor conocido en el ensayo.

El valor de la fuerza resultante sobre el cilindro, $\mathrm{F}_{\mathrm{c}}$, se determina a partir de la ecuación (3):

$$
F_{c}=\sqrt{F_{\rho}^{2}+\left(F_{d}+F_{r c}\right)^{2}}
$$

En función de valores conocidos o medidos en el ensayo, y teniendo en cuenta la ecuación (2), $F_{c}$ se puede expresar conforme a la ecuación (4). Según esto, la determinación de $\mathrm{F}_{c}$, no supone en ningún caso establecer hipótesis de tensiones en el contacto chapa-cilindro a diferencia de lo establecido en ${ }^{[12]}$.

$$
F_{C}=\sqrt{F_{\rho}^{2}+\left(F_{E D E}-F_{E}\right)^{2}}
$$

La fuerza de rozamiento en el cilindro, $\mathrm{F}_{\mathrm{rc}}$, se determina en función del coeficiente de rozamiento, $\mu_{c}$, conforme a lo indicado en la ecuación (5):

$$
\mathrm{F}_{\mathrm{rc}}=\mu_{\mathrm{c}} \cdot \mathrm{F}_{\mathrm{c}}
$$

El coeficiente de rozamiento es función de la presión de contacto, siendo la función de correlación conocida a partir de investigaciones realizadas previamente ${ }^{[15]}$, en las que se demuestra que en el caso estudiado el coeficiente de rozamiento es poco dependiente de la presión de contacto aparente, $\mathrm{P}_{c}$, que se determina conforme a lo indicado en la ecuación (6) a partir de la anchura de la banda de rozamiento, $h_{c}$, y de la longitud del arco de contacto chapa-cilindro, $b_{c}$.

$$
P_{c}=\frac{F_{c}}{h_{c} \cdot b_{c}}
$$

En los diferentes ensayos realizados se ha comprobado que la anchura de la banda de rozamiento producida es muy uniforme, y coincide con la anchura de la chapa. Se ha considerado, por tanto, un valor de $h_{c}$ correspondiente a la anchura de la chapa a su paso por el cilindro de doblado, constante de valor 10,7 mm. La determinación de la longitud del arco de contacto se ha realizado mediante la evaluación directa de la zona de contacto chapa-cilindro, por observación de la misma y contraste con las marcas de lubricante en el cilindro. La medición de la cuerda correspondiente permite establecer la longitud de contacto teniendo en cuenta el radio del cilindro, $R_{c}$, empleado en el ensayo.

La determinación de $\mathrm{P}_{\mathrm{c}}$ a partir de la ecuación (6) supone considerar que la tensión de contacto chapacilindro se distribuye uniformemente, obviando los picos de presión existentes a la entrada y salida del cilindro, conforme a lo determinado por otros autores ${ }^{[7-9]}$. No obstante, los picos de presión máximos corresponden al momento en el que se inicia el doblado, de igual modo en el desdoblado correspondiente, en el radio de entrada a la cavidad de la matriz en el conformado del material. Cuando progresa el conformado la distribución de tensiones, que sigue mostrando valores mayores a la entrada y salida del radio de doblado, presenta valores mucho más uniformes.

En el proceso de embutición profunda, el doblado de la chapa al inicio de la entrada de la cavidad de la matriz no es crítico puesto que la deformación practicada en el prensachapas es mínima. Por tanto, es mucho más realista considerar una distribución de tensiones en el contacto con la forma indicada en la figura $3 \mathrm{c}$ ). 

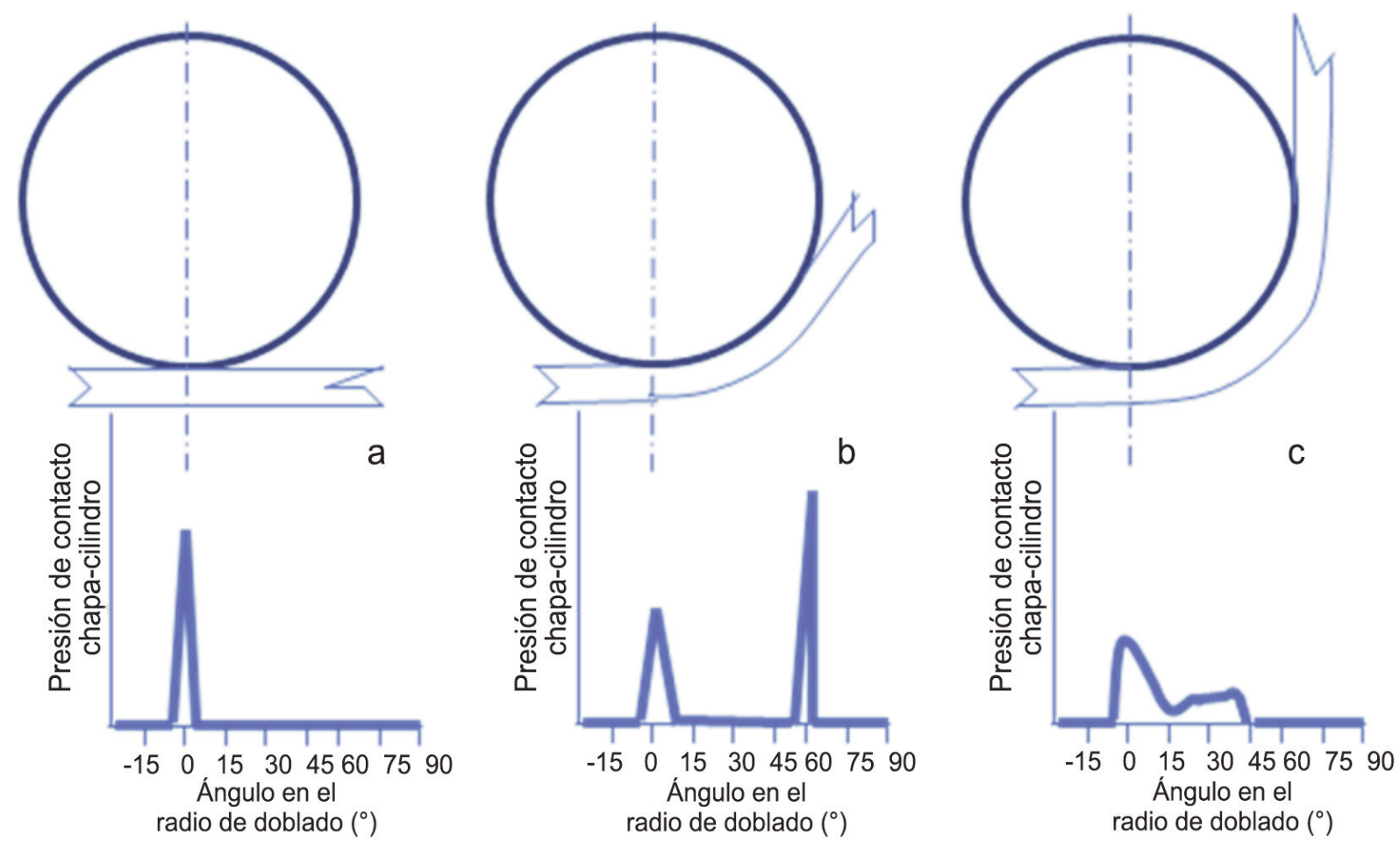

Figura 3. Distribución de presiones en función del ángulo de doblado. a) Inicio de doblado. b) En un punto intermedio del proceso. c) Al final del doblado ${ }^{[7]}$.

Figure 3. Pressure distribution in sheet-bending tool contact as a function of the bending angle. a) Bending begins. b) During the bending process. c) At the end of the bending process ${ }^{[7]}$.

Teniendo en cuenta, además, que la presión no influye significativamente en el coeficiente de rozamiento para el lubricante empleado, se puede considerar como válida la ecuación (6) en lo relativo a la determinación de una presión aparente media en el contacto.

Una vez determinada $F_{r c}$, la fuerza de doblado, $F_{d}$, que incluye en realidad la de doblado y de desdoblado a la entrada y salida del cilindro durante el proceso de deslizamiento de la chapa, se determina a partir de la ecuación (7):

$$
F_{d}=F_{E D E}-F_{E}-F_{r C}
$$

\section{RESULTADOS Y DISCUSIÓN}

\subsection{Determinación de las correlaciones existentes entre las tensiones de ensayo $\sigma_{E D E}$ y $\sigma_{p}$ con el grado de deformación. Influencia del ángulo y del radio de doblado}

El valor de la fuerza de estirado en el ensayo, $\mathrm{F}_{\mathrm{EDE}}$, ha sido normalizado dividiéndolo por la sec- ción transversal de la chapa y, de este modo considerado como tensión, $\sigma_{\mathrm{EDE}}$ Análogamente, la fuerza de reacción medida sobre el cilindro, $\mathrm{F}_{\rho}$, es dividida por el módulo resistente de la chapa, $\mathrm{W}$, para una sección rectangular de anchura $\mathrm{h}_{1} \mathrm{y}$ de espesor, t. El parámetro geométrico $\mathrm{W}$ es obtenido conforme a la expresión (8). El valor normalizado obtenido a partir de $\mathrm{F}_{\rho}$ es simbolizado como $\sigma_{\rho}$.

$$
W=\frac{h_{1} \cdot t^{2}}{6}
$$

En la figura 4 se han representado los valores experimentales de la tensión total de estirado, $\sigma_{\mathrm{EDE}}$ frente a la deformación, $\varepsilon_{\mathrm{h}}$, para los dos radios de doblado experimentados y para los tres ángulos de doblado considerados.

El valor de estos resultados nos muestra la influencia del ángulo de doblado sobre la relación existente entre la fuerza de estirado y la deformación en anchura, observándose claramente que la tensión de estirado es directamente proporcional a la relación de embutición e inversamente proporcional al ángulo de doblado. 

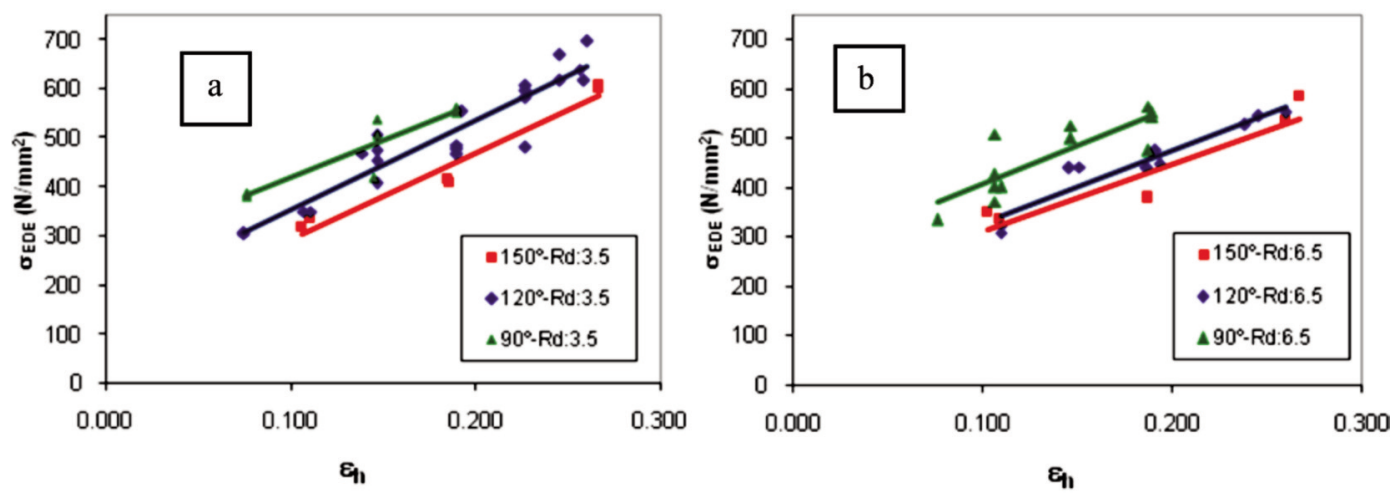

Figura 4. Variación de la tensión de ensayo con la deformación. Ángulos de doblado $150^{\circ}, 120^{\circ}$ y $90^{\circ}$ : a) Radio de doblado $3,5 \mathrm{~mm}$. b) Radio de doblado $6,5 \mathrm{~mm}$.

Figure 4. Stress of the bending test as a function of the deformation. Bending angle values of $150^{\circ}, 120^{\circ}$ and $90^{\circ}$. a) Bending radius $3.5 \mathrm{~mm}$. b) Bending radius $6.5 \mathrm{~mm}$.

Para determinar la influencia del radio de doblado con más detalle se han representado los valores de la tensión de estirado, para una misma relación de embutición, en función del ángulo de doblado (Fig. 5).

Si comparamos los valores de la tensión de estirado obtenidos se observa que para relaciones de embutición inferiores a 0,1 la fuerza de estirado es independiente del radio de doblado. Sin embargo, para relaciones de embutición superiores la fuerza de estirado es mayor cuanto menor es el radio de doblado.

Por tanto, podemos concluir que la tensión de embutición es directamente proporcional a la deformación en anchura, e inversamente proporcional al ángulo y radio de doblado para relacio- nes de deformación significativas, superiores a 0,12 .

Las figuras 6 y 7 muestran los valores experimentales de la fuerza unitaria medida en la bisectriz del ángulo, $\mathrm{F}_{\mathrm{p}} / \mathrm{W}$, en función de la deformación, $\varepsilon_{\mathrm{h}}$, y del ángulo de doblado, respectivamente.

Existe una analogía de comportamiento con lo observado para la tensión de ensayo (Figs. 4 y 5). Estos resultados son lógicos, ya que la reacción en el cilindro es una consecuencia de la fuerza de estirado efectuada en el ensayo y permite establecer la precisión de las mediciones efectuadas en la determinación experimental de $F_{\rho}$.
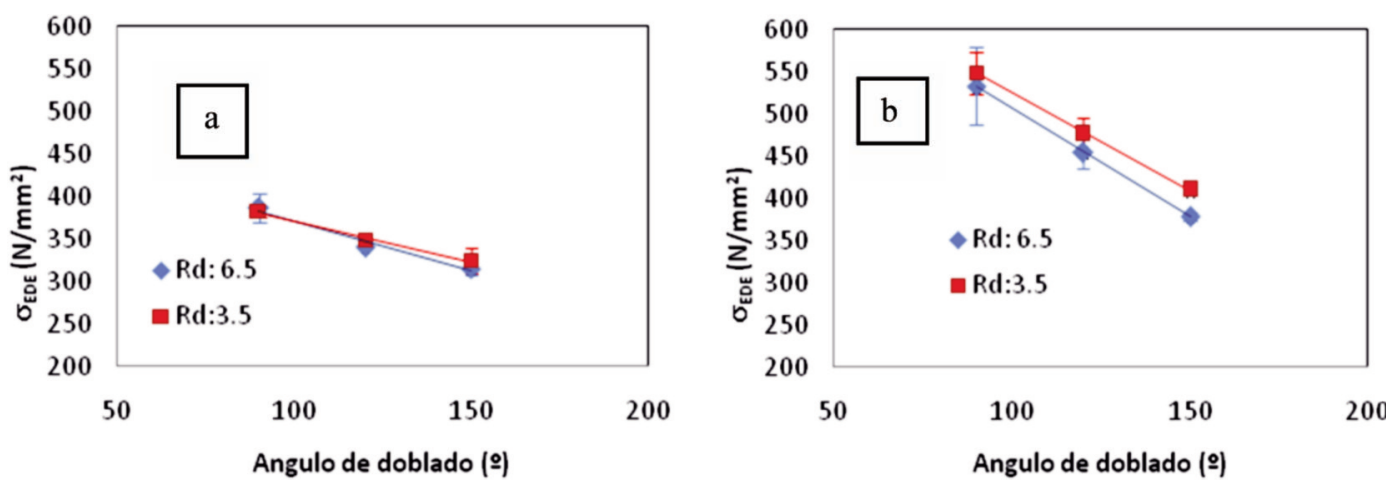

Figura 5. Variación de la tensión de ensayo con el ángulo de doblado para diferentes deformaciones $\varepsilon_{\mathrm{h}}$ a) $\varepsilon_{\mathrm{h}}: 0,11$ b) $\varepsilon_{\mathrm{h}}: 0,19$.

Figure 5. Stress of the bending test as a function of bending angle for different sheet deformation $\varepsilon_{h}$. a) $\varepsilon_{h} 0.11$ b) $\varepsilon_{h}: 0.19$. 

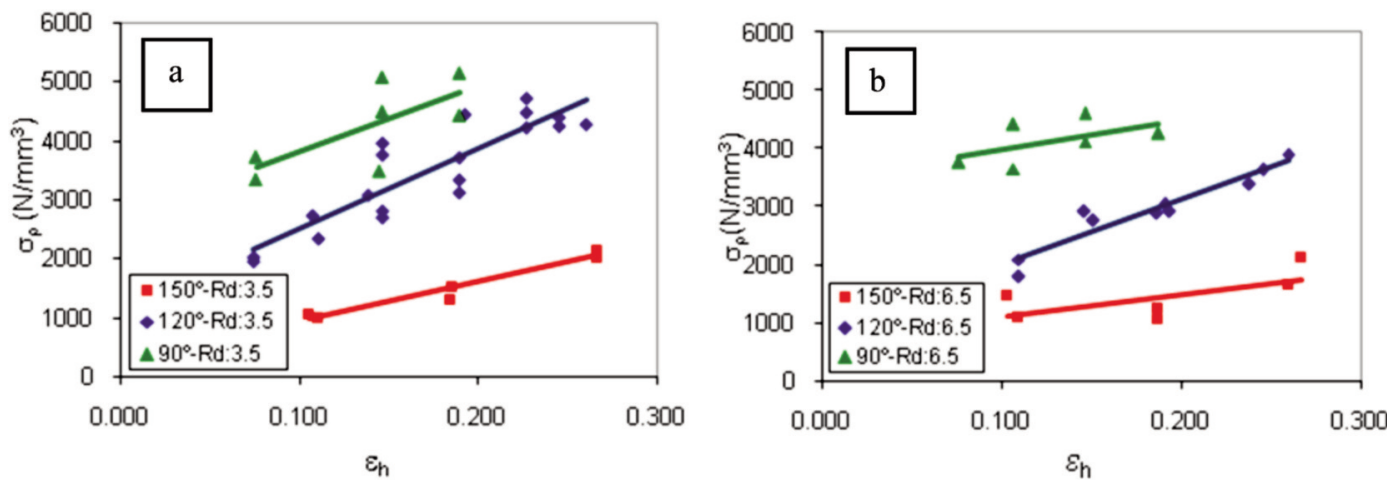

Figura 6. Variación de la fuerza unitaria en la bisectriz del ángulo de doblado, $\sigma_{\rho}$, con respecto a la deformación, $\varepsilon_{\mathrm{h}}$. Ángulos de doblado $150^{\circ}, 120^{\circ}$ y $90^{\circ}$ : a) Radio de doblado 3,5 mm. b) Radio de doblado 6,5 mm.

Figure 6. Normalized sheet-bending tool contact force, $\sigma_{\rho}$, vs. pure shear deformation, $\varepsilon_{h}$. Bending angles $150^{\circ}, 120^{\circ}$ y $90^{\circ}$ : a) Bending radius $3.5 \mathrm{~mm}$. b) Bending radius $6.5 \mathrm{~mm}$.
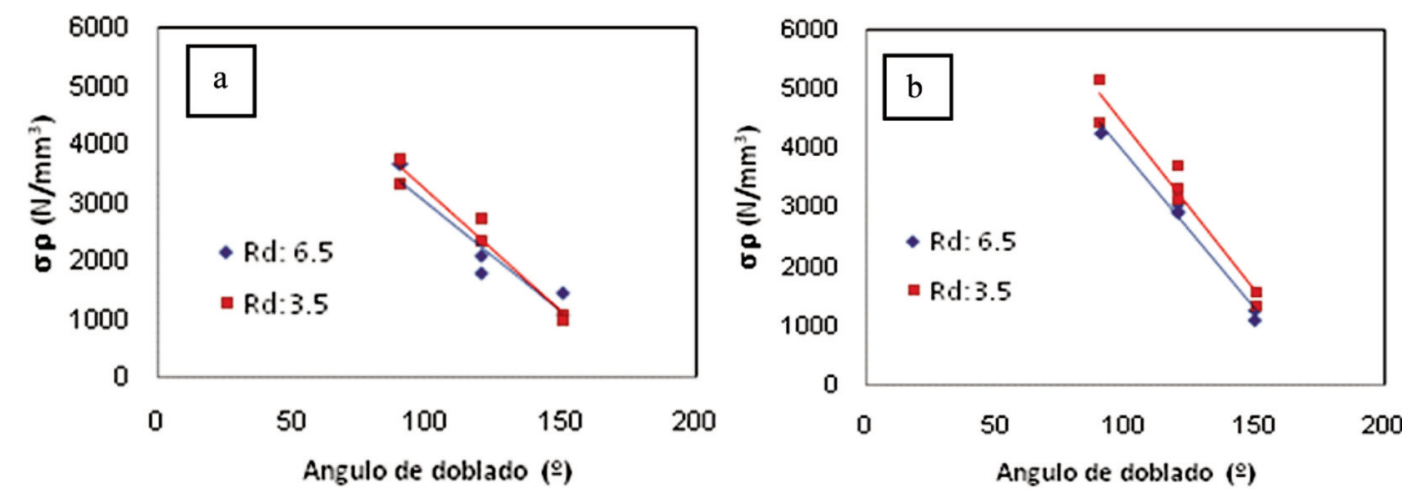

Figura 7. Variación de la fuerza unitaria en la bisectriz, $\sigma_{\mathrm{p}}$, con el ángulo de doblado. Rd 6,5 y $3,5 \mathrm{~mm}$. a) $\varepsilon_{\mathrm{h}}: 0,11$, b) $\varepsilon_{\mathrm{h}}: 0,19$.

Figure 7. Normalized sheet-bending tool contact force, $\sigma_{\rho}$, vs. bendign angle. $R_{d} 6,5$ and $3,5 \mathrm{~mm}$. a) $\varepsilon_{h}: 0.11$, b) $\varepsilon_{h}: 0.19$.

\subsection{Variación de la presión aparente en el cilindro con la deformación en anchura. Influencia del ángulo y del radio de doblado}

En la figura 8 aparecen representados los valores de la presión en el cilindro determinados mediante las ecuaciones (2-6) frente a la deformación en anchura para los diferentes ángulos y radios de doblado. Se observa que la variable principal en este caso es el radio de doblado, siendo la influencia del ángulo muy poco significativa.

En la figura 9 se han representado los valores de $\mathrm{P}_{\mathrm{c}}$ frente al ángulo de doblado para una relación de deformación media de 0,19 , en la que se observa claramente que el radio de doblado tiene una influencia muy significativa sobre la presión de contacto en los tres ángulos de doblado.

Para el radio de doblado de 3,5 la presión es mínima en ángulos de $90^{\circ}$, siendo del mismo orden para ángulos de $120^{\circ}$ y $150^{\circ}$. La fuerza de doblado, tal y como se indica posteriormente en la figura 10 , crece con el ángulo de doblado y, del mismo modo, la fuerza de contacto cilindro-chapa.

Los resultados obtenidos para la presión se justifican porque el arco de contacto chapa-herrramienta disminuye rápidamente con el ángulo de doblado, conduciendo a presiones mayores para los ángulos menores. 


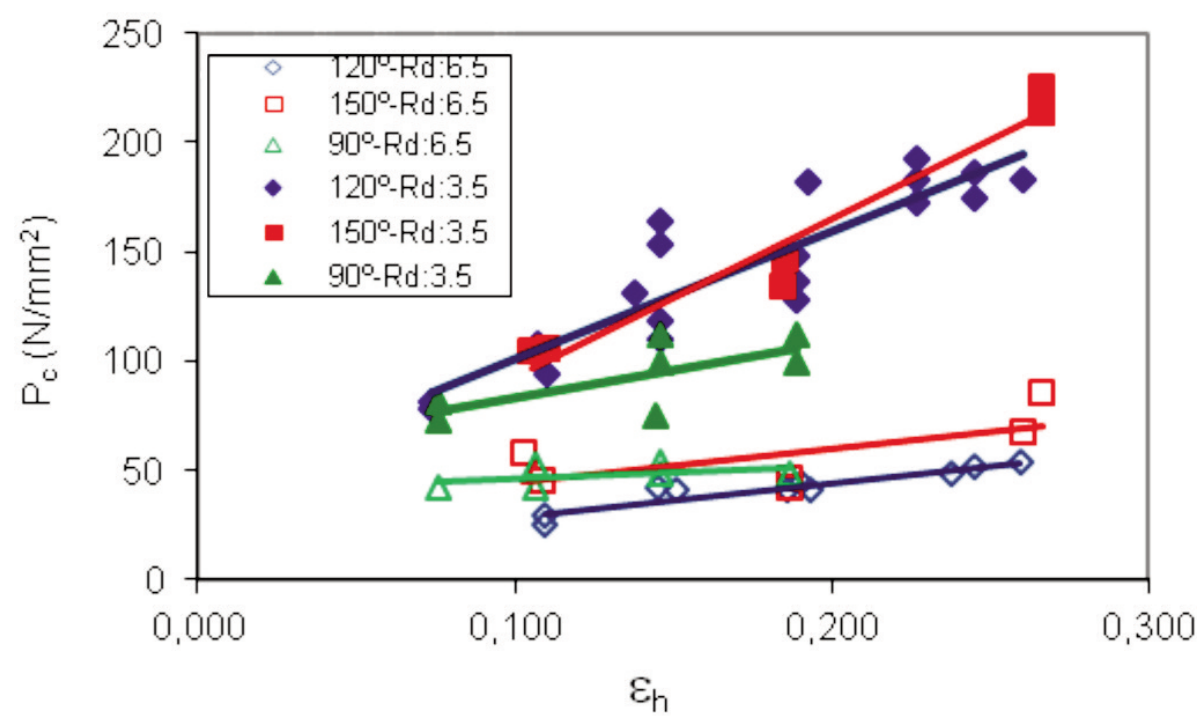

Figura 8. Variación de la presión aparente en el cilindro con la deformación en anchura del material para un ángulo de doblado de $150^{\circ}$, $120^{\circ}$ y $90^{\circ}$. Radios de doblado, $R_{d} 6,5 \mathrm{~mm}$ y $3,5 \mathrm{~mm}$.

Figure 8. Apparent pressure of sheet-tool bending contact vs. material deformation for bending angles of $150^{\circ}, 120^{\circ}$ and $90^{\circ}$. Bending radii of $R_{d} 6.5 \mathrm{~mm}$ and $3.5 \mathrm{~mm}$.

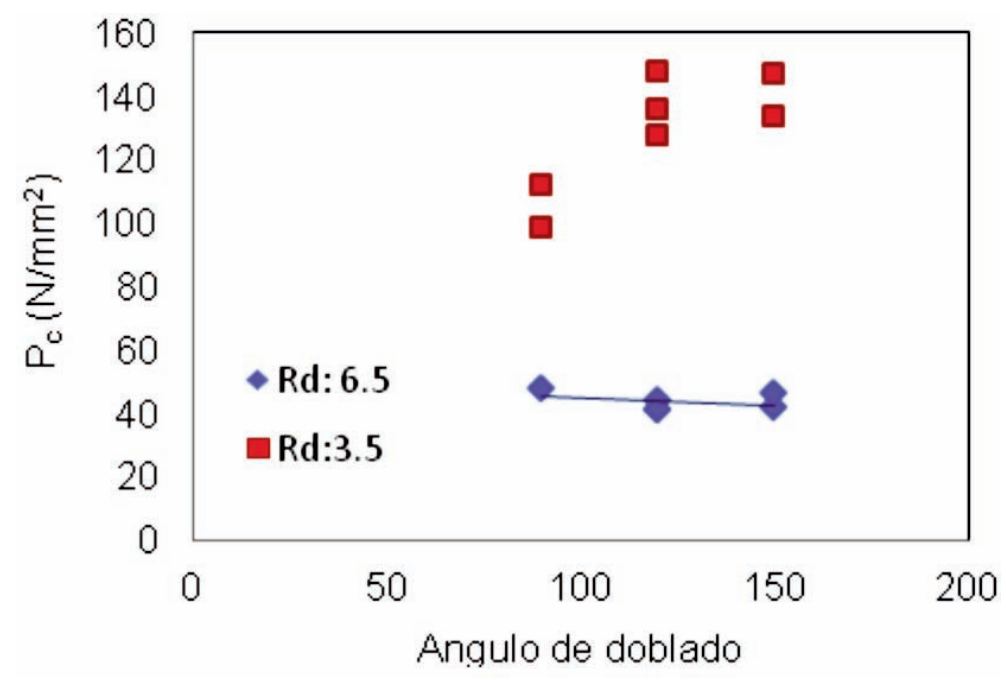

Figura 9. Variación de la presión aparente con el ángulo de doblado para una deformación $\varepsilon_{\mathrm{h}} 0,19$. Radios de doblado $6,5 \mathrm{~mm}$ y $3,5 \mathrm{~mm}$.

Figure 9. Apparent pressure of sheet-bending tool contact vs. bending angle for a deformation $\varepsilon_{h}$ 0.19. Bending radii of $6,5 \mathrm{~mm}$ and $3,5 \mathrm{~mm}$.

Si comparamos los valores de presión alcanzados en estos ensayos, $250 \mathrm{MPa}$, con los obtenidos por otros autores ${ }^{[4,5,7}$ y 8$]$ en diversos procesos de conformado, se observa que en los ensayos realizados en el presente trabajo se alcanzan presiones muy superiores a las obtenidas en los ensayos de doblado bajo tensión existentes en la literatura y son más realistas en relación a los procesos de embutición de chapa. 
La presión aparente tiene una influencia directa en los procesos de fricción en general. Queda demostrado que las presiones en el radio de entrada a la matriz pueden alcanzar valores elevados con rotura de película lubricante y paso de régimen de lubricación mixto a lubricación límite e, incluso, a la rotura de capa adsorbida en este último régimen con adhesiones en el deslizamiento ${ }^{[17]}$. Por tanto, los resultados obtenidos demuestran que el radio de doblado actúa como variable importante en la fricción del proceso de doblado, en tanto que el ángulo de doblado es una variable menos significativa.

\subsection{Determinación de la fuerza de rozamiento y de la fuerza de doblado en el cilindro}

Para la determinación del rozamiento en el cilindro, se va a considerar un coeficiente de rozamiento de 0,08 , determinado previamente en ensayos de rozamiento con contacto plano ${ }^{[15]}$. Aunque en los ensayos indicados se alcanzaron presiones inferiores a $25 \mathrm{MPa}$, se observa que en el rozamiento en el cilindro de doblado la película de lubricante es efectiva, sin existencia de gripado ni adhesiones sobre el mismo. Dado que no existe influencia de la presión sobre el coeficiente de rozamiento en el intervalo de presiones ensayadas ${ }^{[15]}$, se ha establecido como adecuado el valor indicado para el coeficiente de rozamiento en todo el intervalo de presiones correspondientes al contacto chapa-cilindro en el proceso de doblado.
Conforme a las ecuaciones (2-7) se ha determinado la fuerza de doblado en todos los casos experimentados. Los resultados se expresan de forma unitaria, $\sigma_{\mathrm{d}}$, dividiendo el valor de la fuerza por el módulo resistente, W, de la sección de chapa. De todos modos, la anchura y espesor del material permanecen constantes para todos los ensayos.

En la figura 10 aparecen representados los valores obtenidos experimentalmente para la fuerza de doblado en función del ángulo.

Como puede observarse, la fuerza de doblado disminuye conforme el ángulo de doblado aumenta, esto es, cuando el ángulo de doblado es menos exigente, tal y como cabía esperar. El radio de doblado tiene, en cambio, una influencia prácticamente despreciable, a diferencia de lo que ocurre con la fricción en el proceso.

Existen métodos analíticos de cálculo que determinan la fuerza de doblado en función de la geometría del proceso y de las fuerzas que actúan a la entrada y salida del ángulo de doblado en el sentido de deslizamiento de la chapa ${ }^{[16]}$. En las figuras 11 y 12 se representan los resultados obtenidos de forma experimental y analítica para los valores de la fuerza de doblado en función de la presión aparente media en el cilindro. Se ha considerado que el espesor de la chapa no varía durante el doblado, cuestión ésta comprobada experimentalmente.

Por último, se ha establecido una tensión de fluencia para el material constante durante todo el proceso y correspondiente en valor al estado de deformación, $\varepsilon_{\mathrm{h}}$, experimentado por el material.

En ambas figuras se observa que los datos experimentales de la tensión de doblado se ajustan a una

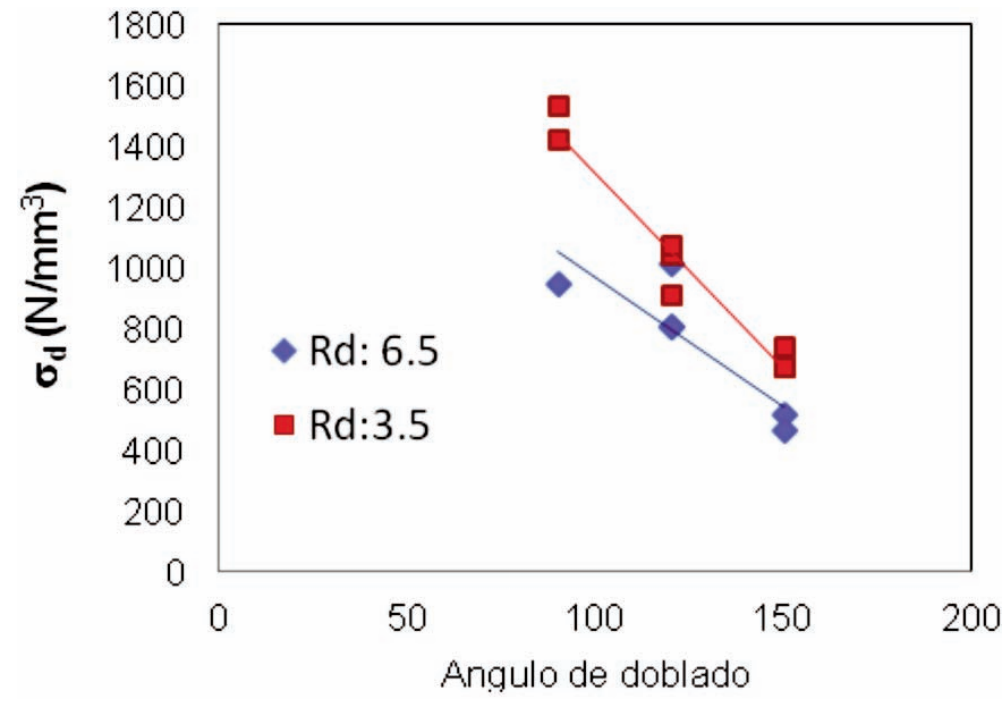

Figura 10. Variación de la tensión de doblado con el ángulo de doblado.

Figure 10. Normalized bending force vs. bending angle. 


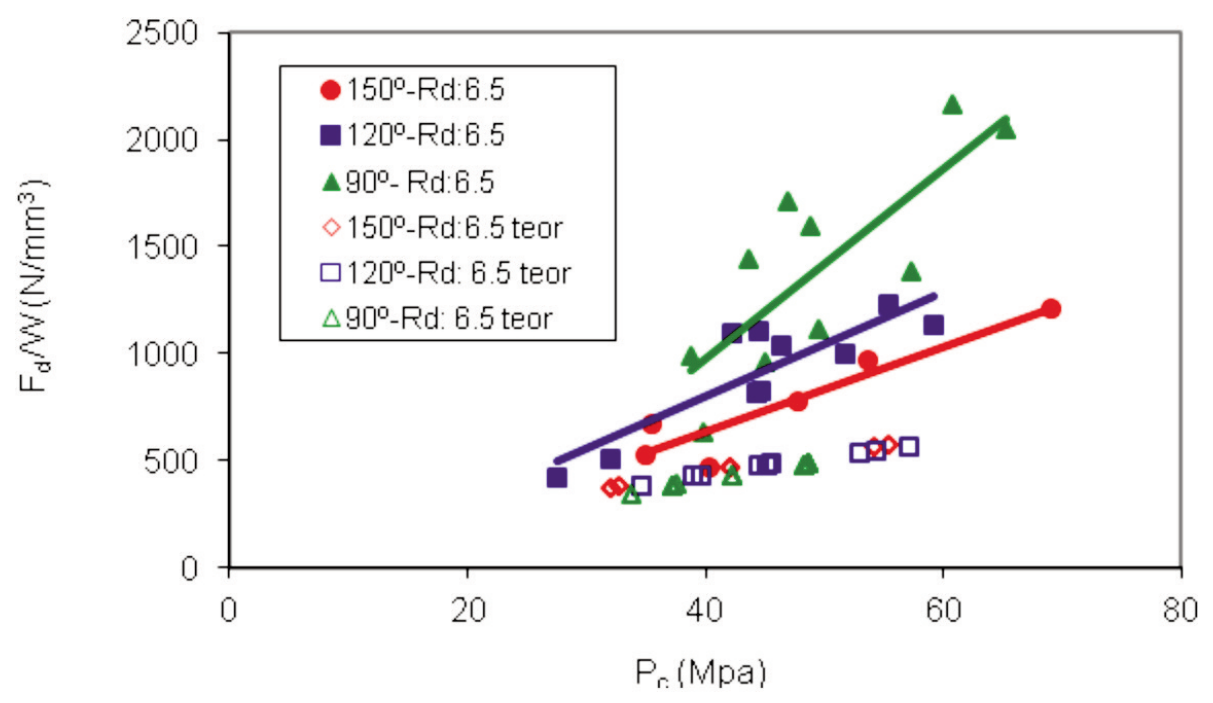

Figura 11. Variación de la tensión de doblado en función de la presión en el cilindro. $R_{d} 6.5 \mathrm{~mm}$.

Figure 11. Normalized bending force vs. apparent pressure in bending contact. $R_{d} 6,5 \mathrm{~mm}$.

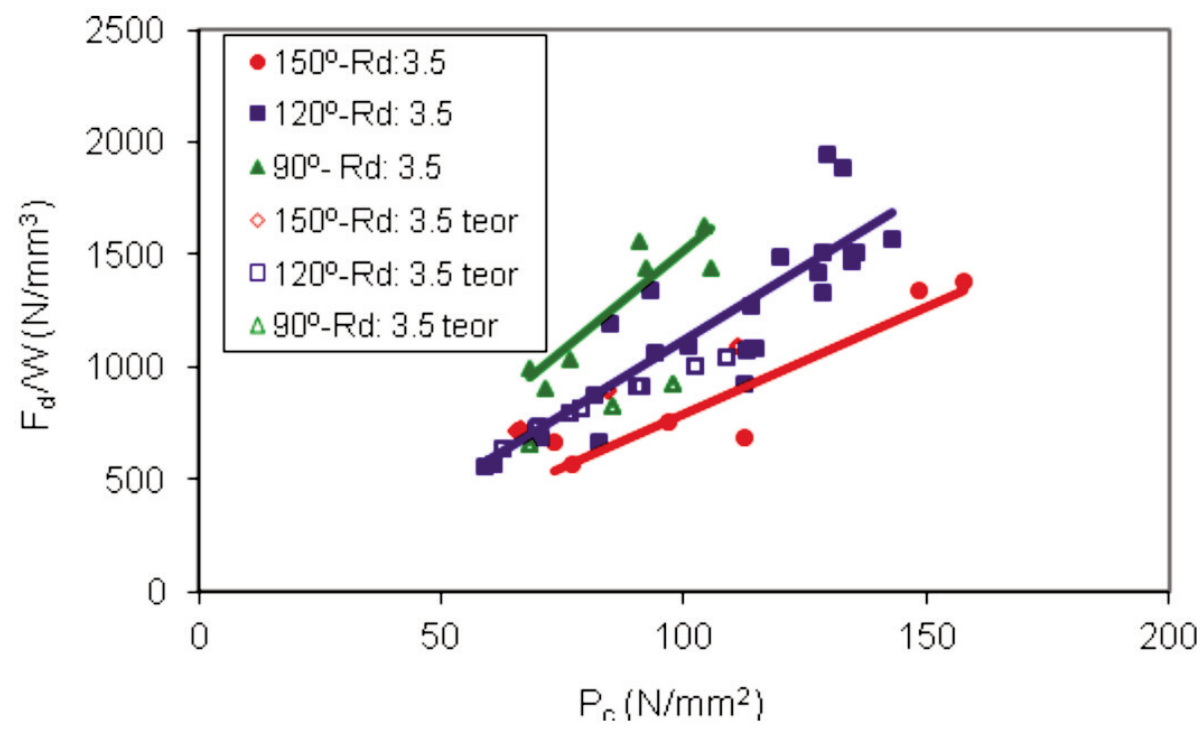

Figura 12. Variación de la fuerza de doblado en función de la presión en el cilindro. Rd: 3,5 mm.

Figure 12. Normalized bending force vs. apparent pressure in bending contact. $R d$ 3,5 $\mathrm{mm}$.

función lineal creciente con la presión en el cilindro, para cada uno de los ángulos y radios estudiados. Este resultado es coherente con el proceso de doblado bajo tensión ya que una mayor presión de contacto es el resultado de mayores fuerzas a la entrada y a la salida del ángulo de doblado y, en consecuencia, la fuerza de doblado debe incrementarse. También se observa que las tensiones de doblado obtenidas son mayores conforme el ángulo de doblado es más exigente. El radio de doblado influye sustancialmente en el valor de la presión de contacto, tal y como se ha comentado con anterioridad. Así, aunque las condiciones de ensayo impuestas por la deformación en el material, $\varepsilon_{\mathrm{h}}$, son similares en el intervalo experimentado, las presiones de contacto en el cilindro son muy superiores con el radio de doblado menor. No obstante, 
se observan tendencias de comportamiento similares con ambos radios de doblado.

La comparación de los resultados obtenidos con los correspondientes al acero dulce DC-0 $5^{[12]}$, con un índice de endurecimiento menor, permiten establecer que la acritud que experimenta el acero 304 DDQ en el proceso de doblado tiene un efecto muy importante. Efectivamente, aunque la tendencia creciente de la tensión de doblado en un acero DC-05 es observada para el ángulo de $90^{\circ}$, se puede establecer que las tensiones de doblado son constantes para ángulos de doblado menos exigentes, $120^{\circ}$ y $150^{\circ}$, lo que significa que el incremento de las tensiones a la entrada y salida del ángulo de doblado tienen una influencia pequeña en el proceso. El radio de doblado tampoco presenta influencia en el caso del acero DC-05. Lógicamente, la pendiente de la función de doblado a $90^{\circ}$ para el acero inoxidable, con un coeficiente de endurecimiento mayor, es también más elevada que para un acero DC-05.

Las tensiones de doblado obtenidas analíticamente son independientes del radio de doblado dado que el método analítico solo considera esta variable para determinar la fricción existente en el cilindro de doblado, e incrementar en el valor correspondiente la tensión a la salida de la chapa ${ }^{[16]} \mathrm{y}$, tal como se observa para las condiciones consideradas, esta cuestión resulta ser despreciable. Los valores obtenidos analíticamente suponen, en prácticamente todos los casos, un límite inferior a los obtenidos experimentalmente, con lo que puede considerarse el método teórico con valor puramente estimativo. La influencia del coeficiente de endurecimiento del material se pone de nuevo de manifiesto. Las funciones analíticas se ajustan mejor a los valores obtenidos para aceros con bajo coeficiente de endurecimiento ${ }^{[14]}$. Por tanto, el análisis efectuado demuestra que la formulación analítica requiere establecer correcciones en función del coeficiente de endurecimiento del material, cuestión ésta que afecta no solo a procesos de doblado bajo tensión sino a otros procedimientos de doblado ${ }^{[17]}$.

\subsection{Consideraciones al ensayo de doblado bajo tensión en dos etapas. Determinación de la fuerza de rozamiento en el cilindro}

Con objeto de establecer el rango de validez del ensayo bajo tensión en dos etapas para la determinación de la fuerza de rozamiento en el proceso de doblado, se han acoplado cilindros giratorios al dispositivo experimental de ensayos, correspondientes a dos de los radios de doblado considerados en el presente trabajo. El cilindro gira sobre un eje de acero templado convenientemente lubricado con la mezcla aceite mineral/ $\mathrm{MoS}_{2}$ al $50 \%$ empleada para la lubricación de las chapas. Según esto, la fuerza de rozamiento en el doblado, $\mathrm{F}_{\mathrm{rc}}$, se determina como diferencia de la fuerza de ensayo en la etapa de rodillo fijo y de rodillo móvil, $\mathrm{F}_{\mathrm{EDE} 1}$ y $\mathrm{F}_{\mathrm{EDE} 2}$, respectivamente.

Los ensayos en dos etapas han sido realizados para un solo ángulo de doblado $\alpha$, de $150^{\circ}$. Los valores de la acción mecánica total en el cilindro de doblado,

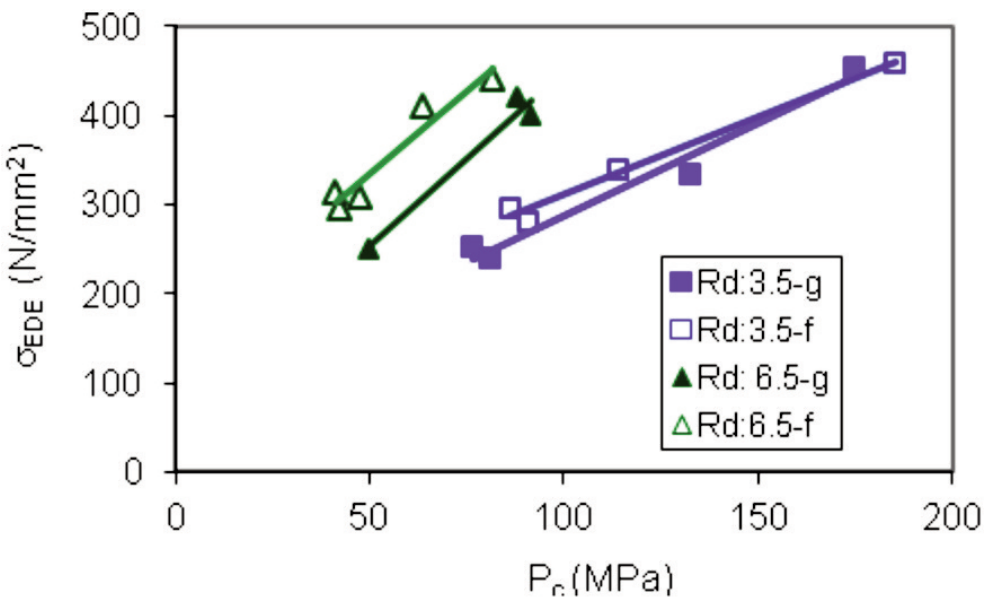

Figura 13. Variación de la tensión de ensayo con respecto a la presión aparente en el cilindro para diferentes radios de doblado con cilindros fijos, $\mathrm{f}$, y giratorios, $\mathrm{g}$.

Figure 13. Normalized bending force vs. apparent pressure in sheet-bending tool contact for differente bending radii and with fixed, $f$, and rolling, $g$, cylinders. 
esto es, la suma de la fuerza de rozamiento y de la de doblado, convenientemente normalizada, se establece gráficamente en la figura 13.

La valoración de estos resultados nos indica que para presiones aparentes de $100 \mathrm{MPa}, \mathrm{R}_{d} 6,50 \mathrm{~mm}$, existen diferencias coherentes entre las tensiones de ensayo obtenidas para un cilindro fijo y giratorio, siendo mayores las del cilindro fijo, tal y como cabría esperar. Sin embargo, para presiones superiores a $100 \mathrm{MPa}, \mathrm{R}_{\mathrm{d}} 3,47 \mathrm{~mm}$, los valores $\sigma_{\mathrm{EDE}}$ son muy similares para ambos cilindros, aún cuando la diferencia de valores debería ser mayor para este último valor del radio de doblado. Según esto, queda demostrado que este tipo de ensayo en dos etapas es solamente aplicable a operaciones que impliquen bajas tensiones de estirado sobre el material, esto es, para bajas presiones aparentes, lo que no suele tener aplicación en procesos reales de conformado. Cuando las fuerzas de estirado son elevadas, superando el límite elástico del material, la tensión de rodadura del cilindro móvil puede adoptar valores que hacen inviable establecer la hipótesis de rozamiento nulo en el cilindro giratorio.

\section{CONCLUSIONES}

- Se han efectuado ensayos de doblado bajo tensión de un acero AISI 304 DDQ. El acero se ha ensayado bajo condiciones de deformación a cortante similar a la que tiene lugar en la zona del prensachapas en los procesos de embutición. En el proceso de estirado se producen de manera simultánea el doblado del material y la deformación previa indicada. Por este motivo, las tensiones involucradas en el ensayo son superiores al límite elástico del material y próximas a la resistencia a tracción de dicho material. De este modo, las tensiones de estirado, así como las presiones de contacto cilindro de doblado-chapa obtenidas reproducen las condiciones existentes en el radio de entrada a la matriz.

- Se han medido experimentalmente las fuerzas involucradas en el ensayo, así como la fuerza de contacto cilindro-chapa en la bisectriz del ángulo de doblado. A partir de dichos valores se obtiene la presión aparente en el cilindro y la tensión de doblado para diferentes deformaciones y ángulo de doblado para dos radios del cilindro diferentes. Se puede establecer que ángulos de doblado crecientes conducen a presiones aparentes mayores, esto es, ángulos menos exigentes de doblado establecen presiones aparentes mayores debido a que la superficie de contacto es relativamente mucho menor para éstos. La variable de influencia más significativa sobre la fuerza de doblado es el radio de doblado, teniendo un efecto menor el ángulo de doblado.
- En el caso de cilindros móviles, la presión aparente cilindro-chapa permite determinar las condiciones de rozamiento en la zona de doblado y predecir el régimen de lubricación para diferentes lubricantes y velocidades de embutición.

\section{REFERENCIAS}

[1] D.P. Kotchman, I. Kim, C.-Y. Sa, y D. Lee, J. Mater. Eng. Perform. 4 (1992) 555-564.

[2] D.W. Vallance y D.K. Matlock, J. Mater. Eng. Perform. 1 (1992) 685-693.

[3] S. Hao, B.E. Klamecki y S. Ramalingam. Wear 224 (1999) 1-7.

[4] A. Azushima, M. Yamamiya y H. Kudo, CIRP Annals Manufacturing Technology 1 (1992) 259-262.

[5] A. Azushima y M. Sakuramoto. CIRP Annals Manufacturing Technology, 1 (2006) 303-306.

[6] P. K. Saha y W. R. D. Wilson, Wear, 172 (1994) 167-173.

[7] M. P. Pereira, J. L. Duncan, W. Yan y B. F. Rolfe, J. Mater. Proc. Tech. 209 (2009) 3.532-3.541.

[8] M. P. Pereira, W. Yan y B. F. Rolfe, Wear 265 (2008) 1.687-1.699.

[9] K. Hanaki y K. Kato, Adv. Technol. Plasticity 1 (1984) 581-586.

[10] G. J. Coubrough, M. J. Alinger y C. J. Van Tyne, J. Mater. Proc. Tech. 130-131 (2002) 69-75.

[11] J. Andreasen, D. Olsson, y K. Chodnikiewicz, Proc. IME B J. Eng. Manufact. 1 (2006) 73-80.

[12] V. Miguel, J. Coello, A. Calatayud, M.C. Manjabacas, A. Martínez y C. Ferrer, J. Mater. Proc. Tech. 1 (2009) 1.588-1.596.

[13] J. Coello, V. Miguel, A. Calatayud, A. Martínez y C. Ferrer, Rev. Metal. Madrid 5 (2010) 435-445.

[14] V. Miguel-Eguía, Investigación de la metodología para la determinación de los indicadores de deformación y de cohesión en tensiones multiaxiales de embutición plana. Aplicación del acero electrocincado DC-05, Universidad Politécnica de Valencia, 2005.

[15] J. Coello, V. Miguel, C. Ferrer, A. Calatayud y A. Martínez, Rev. Metal. Madrid 6 (2008) 503-512.

[16] Z. Marciniak, J.L Duncan y S. J. Hu, Mechanics of sheet metal forming. Ed. ButterworthHeinemann; $2^{\text {nd }}$ ed; UK, 2002.

[17] V. Miguel, J. Coello, M. C. Manjabacas, A. Calatayud, C. Ferrer y A. Martínez, J. Tribol, 1 (2011) 1-9.

[18] FJ. Avellaneda, V. Miguel, J. Coello, A. Martinez y A. Calatayud, IV Manufacturing Engineering Society International Conference, American Institute of Physics (en fase de publicación). 